\title{
Effects of Whole Body Therapeutic Hypothermia on Gastrointestinal Morbidity and Feeding Tolerance in Infants with Hypoxic Ischemic Encephalopathy
}

\author{
Kimberly M. Thornton, ${ }^{1,2}$ Hongying Dai, ${ }^{3}$ Seth Septer, ${ }^{2,4}$ and Joshua E. Petrikin ${ }^{1,2}$ \\ ${ }^{1}$ Department of Neonatology, Children's Mercy Hospital, 2401 Gillham Road, Kansas City, MO 64108, USA \\ ${ }^{2}$ School of Medicine, University of Missouri-Kansas City, 2401 Gillham Road, Kansas City, MO 64108, USA \\ ${ }^{3}$ Research Development and Clinical Investigation, Children's Mercy Hospital, 2401 Gillham Road, Kansas City, MO 64108, USA \\ ${ }^{4}$ Department of Gastroenterology, Children’s Mercy Hospital, 2401 Gillham Road, Kansas City, MO 64108, USA
}

Correspondence should be addressed to Kimberly M. Thornton; kimmcdonaldthornton@gmail.com

Received 4 June 2014; Accepted 25 July 2014; Published 25 August 2014

Academic Editor: Tonse N. K. Raju

Copyright (C) 2014 Kimberly M. Thornton et al. This is an open access article distributed under the Creative Commons Attribution License, which permits unrestricted use, distribution, and reproduction in any medium, provided the original work is properly cited.

Objective. This retrospective cohort study evaluated the effects of whole body therapeutic hypothermia (WBTH) on gastrointestinal (GI) morbidity and feeding tolerance in infants with moderate-to-severe hypoxic ischemic encephalopathy (HIE). Study Design. Infants $\geq 35$ weeks gestational age and $\geq 1800$ grams birth weight with moderate-to-severe HIE treated from 2000 to 2012 were compared. 68 patients had documented strictly defined criteria for WBTH: 32 historical control patients did not receive WBTH (non-WBTH) and 36 cohort patients received WBTH. Result. More of the non-WBTH group infants never initiated enteral feeds ( $28 \%$ versus $6 \% ; P=0.02$ ), never reached full enteral feeds (38\% versus $6 \%, P=0.002)$, and never reached full oral feeds $(56 \%$ versus $19 \%, P=0.002$ ). Survival analyses demonstrated that the WBTH group reached full enteral feeds (median time: 11 versus 9 days; $P=0.02$ ) and full oral feeds (median time: 19 versus 10 days; $P=0.01$ ) sooner. The non-WBTH group had higher combined outcomes of death and gastric tube placement (47\% versus $11 \% ; P=0.001$ ) and death and gavage feeds at discharge (44\% versus $11 \% ; P=0.005)$. Conclusion. WBTH may have beneficial effects on GI morbidity and feeding tolerance for infants with moderateto-severe HIE.

\section{Introduction}

Perinatal HIE is associated with high morbidity and mortality in the neonatal period as well as long-term neurocognitive deficits. Although slightly different inclusion criteria were used among studies, multiple randomized controlled trials demonstrate that WBTH has a statistically significant improvement in neurodevelopmental disability at 18 to 24 months of [1-3] followup and 6 to 7 years of followup [4] for infants with moderate-to-severe HIE at birth. However, the effects of a perinatal hypoxic ischemic event extend beyond the brain and neurodevelopment. Decreased perfusion to the GI tract [5] and decreased motility leading to feeding intolerance [6] may follow perinatal asphyxia. Patients can present with symptoms of GI bleeding, vomiting, diarrhea, and even necrotizing enterocolitis (NEC) following HIE $[7,8]$. Since WBTH acts to prevent secondary damage to the brain from ischemia and reperfusion injury that occurs following periods of perinatal anoxia, it is plausible to believe that WBTH could have similar preventative effects on the ischemic damage to the GI system.

Previous studies demonstrate conflicting results. There were some indications of hepatic dysfunction and feeding disturbances seen in subjects involved in a few of the major studies for therapeutic hypothermia, but none of those studies focused specifically on the effects to the GI system as a primary outcome. For example, the Cool Cap study, using selective head cooling, reported elevated liver enzymes (aspartate transaminase $>200 \mathrm{IU} / \mathrm{L}$ and alanine transaminase $>100 \mathrm{IU} / \mathrm{L}$ ) in $38 \%$ of the cooled subjects versus $53 \%$ of 
controls $(P=0.02)$ [9]. Another selective head cooling trial, performed by Zhou et al., reported raised liver enzymes (not defined) in $35 \%$ of the cooled subjects versus $28 \%$ of the controls $(P>0.05)[10]$. The ICE study reported $35 \%$ of subjects who received WBTH with hepatic dysfunction (alanine aminotransferase level $>100 \mathrm{U} / \mathrm{L}$ ) versus $45 \%$ of controls $(P>0.05)$. This study also found a higher incidence of GI impairment (sloughing of the bowel, rectal bleeding, or NEC) in $4 \%$ of cooled subjects versus $2 \%$ of controls $(P>0.05)[2]$. Another WBTH study, sponsored by the National Institute of Child Health and Human Development (NICHD), reported hepatic dysfunction (aspartate aminotransferase level $>200 \mathrm{IU}$ and alanine aminotransferase level $>100 \mathrm{IU}$ ) in $20 \%$ of cooled subjects versus $15 \%$ of controls (significance not reported but calculated $P>0.05$ ). In the cooled group, $11 \%$ of patients were discharged on gavage feeds versus $7 \%$ of controls (significance not reported but calculated $P>0.05$ ) and $7 \%$ of patients were discharged with a gastric tube (GT) versus $17 \%$ of controls (significance not reported but calculated $P>0.05$ ) [3]. Finally, the TOBY trial for WBTH reported only one case of NEC in a cooled patient and no cases of NEC in the control group ( $<1 \%$ versus $0 \%$, no significance) [1].

To our knowledge, there are no published clinical trials evaluating the effects of therapeutic hypothermia for GI ischemia and reperfusion injury following HIE in humans, but several animal studies suggest a benefit. Pierro and Eaton [11] developed a rat model of intestinal ischemia-reperfusion by surgically isolating and temporarily occluding the superior mesenteric artery for 30-minute duration and then assessing signs of metabolic and histologic damage incurred to the GI tract after 60 minutes of reperfusion. They demonstrated evidence of liver energy failure, intestinal damage, and $100 \%$ mortality in the animals within 4 hours of reperfusion at normothermia. However, when the rats were kept hypothermic $\left(32-33^{\circ} \mathrm{C}\right)$ by controlling the environmental temperature, there was a significant decrease in metabolic and histologic damage as well as $100 \%$ survival [11]. Hassoun et al. used a similar rat model with 45 minutes of induced ischemia and 6 hours of reperfusion to demonstrate decreased levels of inflammatory markers (nuclear factor kappa-B and inducible nitric oxide synthase); an increased level of a protective marker (heme oxygenase-1) and decreased histologic intestinal damage after direct hypothermia was applied to the GI tract during the ischemic period [12]. Finally, Stefanutti and colleagues [13] used the same rat model with "rescue" hypothermia applied by controlling the environmental temperature only during the reperfusion period. After 60 minutes of ischemia and 2 to 5 hours of reperfusion, hypothermia led to $100 \%$ survival and reduced inflammation, metabolic injury, and histologic damage to the GI tract [13].

We wanted to apply this evidence for WBTH use in animal models with ischemia-reperfusion injury to clinical practice. The purpose of our study was to evaluate the effects of WBTH on GI function and feeding tolerance in infants diagnosed with moderate-to-severe HIE. The primary objective was to determine the number of days to reach full enteral feeds and to reach full oral feeds in a group of infants with HIE treated with WBTH compared to a historical control group of infants with HIE prior to the routine use of WBTH. Our hypothesis was that WBTH would have a protective effect on GI function and feeding tolerance status post-moderate-to-severe HIE.

\section{Methods}

2.1. Study Population: Total. Study approval was obtained from the Institutional Review Board of Children's Mercy Hospitals (Kansas City, MO). There were 435 total patients identified for retrospective analysis: 347 in the non-WBTH group and 88 in the WBTH group. 287 total patients were excluded from the study because they did not meet criteria for moderate-to-severe HIE, because they had a significant congenital defect at birth, because they were enrolled in another research study involving WBTH, or because there was insufficient data available in the medical record. There were 78 patients in the non-WBTH general criteria (defined below) group and 70 patients in the WBTH general criteria group. There were 32 patients in the non-WBTH strict criteria (defined below) group and 36 patients in the WBTH strict criteria group (Figure 1).

2.2. Study Population: Non-WBTH Group. Medical charts were reviewed for all infants $\geq 35$ weeks of gestational age and $\geq 1800$ grams of birth weight admitted to our institution from January 2000 to December 2008 with at least one of the following diagnoses on admission: hypoxic ischemic encephalopathy, birth asphyxia, meconium aspiration, nuchal cord, placental abruption, seizure, depression at birth, acidosis at birth, and/or shoulder dystocia. Strict criteria for the use of WBTH at our institution are based on previously published data $[1-3,9,10,14]$ and defined as a history of birth asphyxia, severe metabolic acidosis on cord blood gases or blood gas obtained within 1 hour of life $(\mathrm{pH}$ $\leq 7$ or base deficit $\geq 16$ or $\mathrm{pH} 7.01-7.15$ or base deficit $10-16$ and 10 -minute APGAR score $\leq 5$ or assisted ventilation at birth continued $\geq 10$ minutes), and seizures or other evidence of moderate-to-severe encephalopathy (decreased consciousness, decreased-to-no activity, distal flexion or decerebrate posturing, hypotonia, decreased or absent reflexes, abnormal pupillary response, and abnormal breathing pattern) as documented in the medical record. Patients with adequate documentation were included in the study as historical controls and defined as the non-WBTH strict criteria group. When data for these criteria was missing, the record was reviewed further to identify other evidence of significant HIE. If patients had indirect evidence of moderate-to-severe HIE (abnormal lab values, abnormal neuroimaging, death, etc.), they were included as historical controls in the larger non-WBTH general criteria group. If the degree of HIE was considered to be mild or insufficient evidence was ultimately found to determine HIE status, the patient was excluded from the analysis. Patients with significant congenital anomalies at birth (congenital heart disease, gastroschisis, omphalocele, intestinal atresia, chromosomal trisomy, hydrocephalus, etc.) or patients involved in another study protocol using WBTH were also excluded. 


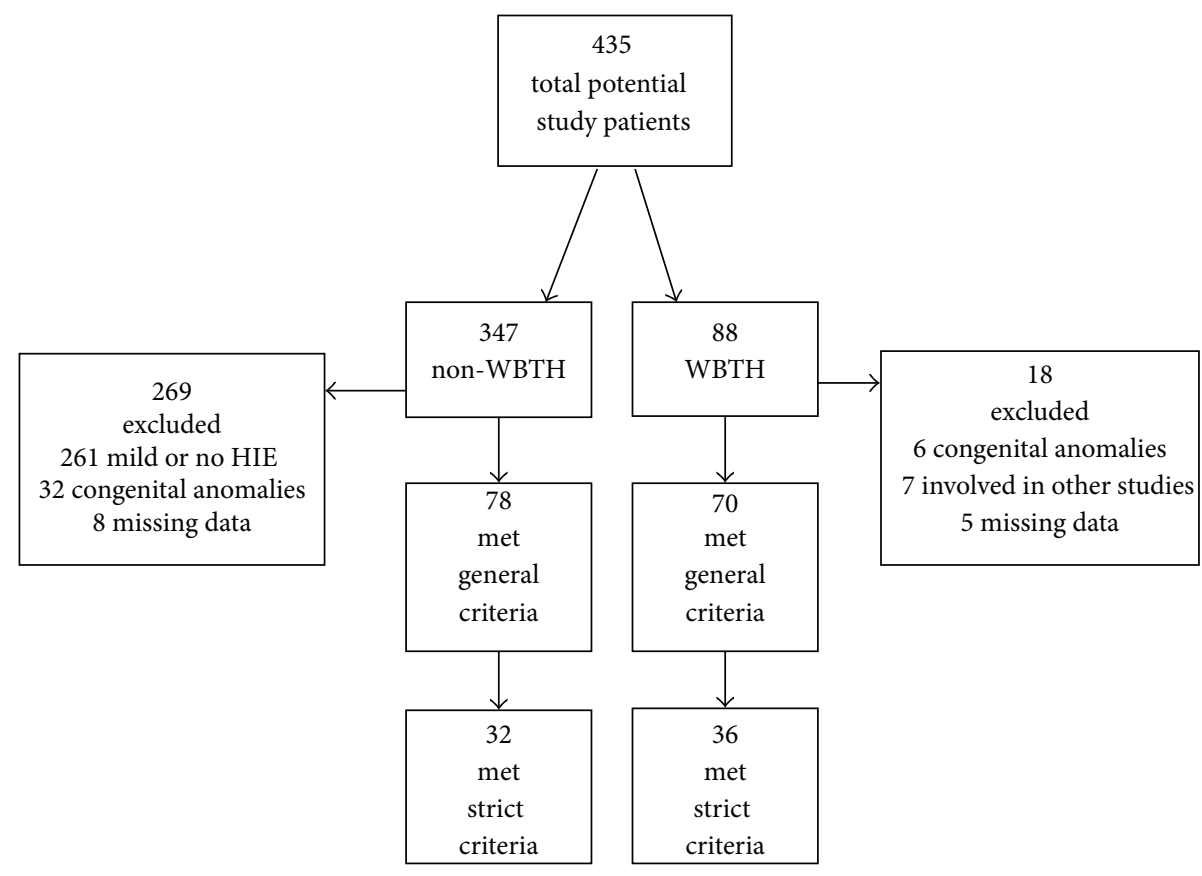

FIGURE 1: Study population.

2.3. Study Population: WBTH Group. A data set had been previously collected for quality purposes on all patients receiving WBTH from the start of standard practice use at our institution in December 2008 up to December 2012. These patients had been diagnosed with moderate-to-severe HIE based on the aforementioned criteria and completed 72 hours of WBTH at $33-34^{\circ} \mathrm{C}$. In some instances, inadequate documentation existed so that all of the criteria were not found for each patient. The WBTH strict criteria group included only those patients with all criteria clearly documented, while the WBTH general criteria group included all patients who received WBTH. Again, patients with significant congenital anomalies at birth (congenital heart disease, gastroschisis, omphalocele, intestinal atresia, hydrocephalus, etc.) or patients involved in another study protocol using WBTH were excluded. Of note, patients with moribund conditions or congenital/chromosomal anomalies with poor likelihood of survival in the neonatal period were not treatment candidates as per the standard protocol for WBTH at our institution.

2.4. Study Design. We performed a twofold analysis of the data by comparing infants in the strict criteria group who received WBTH to those who did not and comparing infants in the general criteria group who received WBTH to those who did not. If information was missing in the medical record for the individual research parameters, the patient was excluded only from the statistical analysis of that specific parameter. Primary outcomes were day of life (DOL) enteral feeds started, DOL full enteral feeds reached, and DOL full oral feeds reached. Secondary outcomes included never started enteral feeds, never reached full enteral feeds, never reached full oral feeds, death, GT, combined death and GT, discharge with gavage feeds, combined death and discharge with gavage feeds, NEC, use of extracorporeal membrane oxygenation (ECMO), elevated liver enzymes, elevated coagulation parameters, and days to hospital discharge or death. These outcomes were chosen as objective measures of GI morbidity and feeding tolerance that could be easily determined from the electronic medical documentation. Other variables such as gastric residuals and withholding of feeds were considered for outcome measures but were not well documented in the electronic medical record and could not be used for this study.

2.5. Statistical Methods. Data are expressed as mean \pm standard deviation (SD) for continuous outcome variables and percentage for categorical outcome variables. Characteristics of WBTH and non-WBTH patients were compared using Chi-square test, Fisher's exact test, and $t$-test. Patients who did not start or reach full enteral feeds due to death were treated as censored data. Patients who did not reach full oral feeds due to death, GT placement, or discharge with gavage feeds were also treated as censored data. Kaplan-Meier survival curves were generated for WBTH and non-WBTH patients. We then performed the log-rank test to compare survival curves between the two groups. All analyses were performed using SAS 9.2 (Cary, NC) and SPSS 20. Statistical significance was claimed at 95\% confidence level $(P<0.05)$.

\section{Results}

3.1. Comparison of Demographic Data. Overall, there were more males (59\% of total study population) than females and more Caesarean sections performed (66\% of total study population) than vaginal deliveries for infants with moderateto-severe HIE. There were slight differences in birth weight, gestational age, and maternal age between the groups, but we do not feel that these differences affected our results. The 
TABLE 1: Demographics for all subjects.

\begin{tabular}{|c|c|c|c|}
\hline Variable & WBTH & Non-WBTH & $P$ value \\
\hline General criteria group & $(n=70)$ & $(n=78)$ & \\
\hline Gender (\% male) & $44(63 \%)$ & $44(56 \%)$ & 0.50 \\
\hline Birth weight (grams) & $3447.1 \pm 524.2$ & $3262.0 \pm 570.3$ & $0.04^{*}$ \\
\hline Gestational age (weeks) & $39.0 \pm 1.5$ & $38.4 \pm 1.9$ & $0.02^{*}$ \\
\hline Maternal age (years) & $25.4 \pm 6.3$ & $29.8 \pm 15.6$ & $0.03^{*}$ \\
\hline Cesarean delivery & $45(64 \%)$ & $52(67 \%)$ & 0.86 \\
\hline 5-minute APGAR score & $3.0 \pm 2.1$ & $3.3 \pm 2.0$ & 0.29 \\
\hline Strict criteria group & $(n=36)$ & $(n=32)$ & \\
\hline Gender (\% male) & $18(50 \%)$ & $18(56 \%)$ & 0.63 \\
\hline Birth weight (grams) & $3510 \pm 523.7$ & $3120.1 \pm 568.6$ & $<0.01^{*}$ \\
\hline Gestational age (weeks) & $39.2 \pm 1.5$ & $38.3 \pm 1.7$ & $0.02^{*}$ \\
\hline Maternal age (years) & $25.4 \pm 6.9$ & $27.9 \pm 7.6$ & 0.17 \\
\hline Cesarean delivery & $21(58 \%)$ & $23(72 \%)$ & 0.31 \\
\hline 5-minute APGAR score & $4.1 \pm 1.8$ & $2.2 \pm 1.8$ & $<0.001^{*}$ \\
\hline
\end{tabular}

* Significance defined by $P<0.05$.

degree of HIE, defined by the 5-minute APGAR score, was similar for the general criteria group, but the strict WBTH group had lower APGAR scores at 5 minutes when compared with the non-WBTH group $(2.2 \pm 8$ versus $4.1 \pm 1.8, P<0.001)$. However, the mean score was below 5 at 5 minutes for both groups, suggesting that the groups had comparable degrees of depression at birth (Table 1).

3.2. Comparison between WBTH $(n=36)$ and Non-WBTH $(n=32)$ Using Strict Criteria. The non-WBTH group had more infants who never started on enteral feeds due to death (28\% versus $6 \%, P=0.02$ ), more infants who never reached full enteral feeds due to death (38\% versus $6 \%, P=0.002)$, and more infants who never reached full oral feeds due to death, GT placement, or discharge with gavage feeds (56\% versus $19 \%, P=0.002$ ) as compared to the WBTH group. The non-WBTH group has a higher mortality rate than the WBTH group (32\% versus 6\%, OR (95\% CI): 7.9 (1.6-39.5), $P=0.009)$. The non-WBTH group had a higher rate of combined death and GT placement (46.9\% versus $11.1 \%, P=$ 0.001 ) and combined death and gavage feeds at discharge (43.8 versus 11.1, $P=0.005$ ). Survival analysis was performed to take the censoring into account. The Kaplan Meier curves demonstrated that infants in the WBTH group reached full enteral feeds (median time: 11 versus 9 days, $P=0.02$ ) and full oral feeds (median time: 19 versus 10 days, $P=0.01$ ) sooner than those in the non-WBTH group (Figure 2). There were only 3 cases of NEC, but they were all in the non-WBTH group ( $9 \%$ versus $0 \%, P=0.1$ ) There was no difference in elevation of liver enzymes (63\% versus $63.9 \%, P=0.94$ ) or abnormal coagulation factors ( $91.3 \%$ versus $100 \%, P=0.15$ ) between groups (Table 2).

3.3. Comparison between WBTH $(n=70)$ and Non-WBTH ( $n=78)$ Using General Criteria. There was no statistical significance between groups for primary outcomes, mortality, or GI morbidity measures. There were few cases of NEC, and there was no statistical significance of occurrence of NEC between groups ( $5 \%$ versus $3 \%, P=0.68$ ). However, there were an increased number of infants who never reached full oral feeds due to death, GT placement, or discharge with gavage feeds in the non-WBTH group versus the WBTH group ( $42 \%$ versus $26 \%, P=0.03$ ). Also, infants who received WBTH had elevated coagulation parameters (prothrombin time $>15.6$ seconds and partial thromboplastin time $>41.5$ seconds) ( $80 \%$ versus $100 \%, P=0.0001)$ more often than those not treated with WBTH, but there was no difference in the number of infants with elevated liver enzymes between groups $(54.6 \%$ versus $62.9 \%, P=0.33$ ) (Table 3 ).

\section{Discussion}

Our analysis suggests that infants treated with WBTH for moderate-to-severe HIE may have improved feeding tolerance, GI morbidity, and overall mortality compared with those not treated with WBTH. Infants treated with WBTH strict criteria group reached full enteral feeds and full oral feeds sooner than those not treated. The WBTH strict criteria group had a lower overall mortality as well as combined GI morbidity (defined as GT placement or gavage feeds at discharge) and mortality.

Our results show that the majority of subjects had elevated liver enzymes and elevated coagulation parameters, expected sequelae of HIE $[15,16]$. Other studies have reported that these effects were only temporary with improvement in liver enzymes and coagulation parameters over time, and our results were consistent (data not shown). There was, however, no significant difference in the number of infants with elevated liver enzymes between the WBTH and nonWBTH groups in our study.

Interestingly, there was no difference in the DOL feeds initiated between groups in either analysis. This retrospective study covered a wide time frame during which many changes occurred in medical practice, including the standard use of WBTH for moderate-to-severe HIE and the use of standardized feeding protocols. The feeding protocols are 
TABLE 2: Primary and secondary outcome variables for strict criteria group.

\begin{tabular}{|c|c|c|c|}
\hline Variable & $\begin{array}{l}\text { WBTH } \\
(n=36)\end{array}$ & $\begin{array}{l}\text { Non-WBTH } \\
\quad(n=32)\end{array}$ & $P$ value \\
\hline \multicolumn{4}{|c|}{ Primary outcomes $($ mean \pm SD) } \\
\hline DOL enteral feeds started & $5.6 \pm 2$ & $6 \pm 3$ & 0.12 \\
\hline DOL full enteral feeds reached & $10.1 \pm 3.7$ & $11.3 \pm 5.6$ & $0.02^{*}$ \\
\hline DOL full oral feeds reached & $11.5 \pm 5.6$ & $12.7 \pm 6.7$ & $0.01^{*}$ \\
\hline \multicolumn{4}{|c|}{ Secondary outcomes (\%) } \\
\hline Never started enteral feeds & 5.6 & 28.1 & $0.02^{*}$ \\
\hline Never reached full enteral feeds & 5.6 & 37.5 & $0.002^{*}$ \\
\hline Never reached full oral feeds & 19.4 & 56.3 & $0.002^{*}$ \\
\hline Death & 5.7 & 32.3 & $0.009^{*}$ \\
\hline GT & 5.6 & 15.6 & 0.24 \\
\hline Combined death and GT & 11.1 & 46.9 & $0.001^{*}$ \\
\hline Discharged with gavage feeds & 5.6 & 9.4 & 0.66 \\
\hline Combined death and discharged with gavage feeds & 11.1 & 43.8 & $0.005^{*}$ \\
\hline $\mathrm{NEC}^{+}$ & 0 & 9.4 & 0.1 \\
\hline ECMO & 8.3 & 0 & 0.24 \\
\hline Elevated liver enzymes ${ }^{\dagger}$ & 63.9 & 63 & 0.94 \\
\hline Elevated coagulation parameters ${ }^{\infty}$ & 100 & 91.3 & 0.15 \\
\hline Days to hospital discharge or death & $16.9 \pm 11.1$ & $16.9 \pm 12$ & 1 \\
\hline
\end{tabular}

* Significance defined by $P<0.05$.

${ }^{+}$Grossly bloody stool (Bell's stage IIA or greater).

${ }^{\dagger}$ Aspartate aminotransferase level $>200$ IU and alanine aminotransferase level $>100$ IU.

${ }^{\infty}$ Prothrombin time $>15.6$ seconds and partial thromboplastin time $>41.5$ seconds.

TABLE 3: Primary and secondary outcome variables for general criteria group.

\begin{tabular}{|c|c|c|c|}
\hline Variable & $\begin{array}{c}\text { WBTH } \\
(n=70)\end{array}$ & $\begin{array}{l}\text { Non-WBTH } \\
\quad(n=78)\end{array}$ & $P$ value \\
\hline \multicolumn{4}{|c|}{ Primary outcomes (mean \pm SD) } \\
\hline DOL enteral feeds started & $5.4 \pm 2.2$ & $5.8 \pm 2.6$ & 0.39 \\
\hline DOL full enteral feeds reached & $9.9 \pm 4.3$ & $10.4 \pm 6.1$ & 0.12 \\
\hline DOL full oral feeds reached & $11.4 \pm 6.2$ & $11.4 \pm 6.9$ & 0.13 \\
\hline \multicolumn{4}{|c|}{ Secondary outcomes (\%) } \\
\hline Never started enteral feeds & 12.9 & 21.8 & 0.15 \\
\hline Never reached full enteral feeds & 12.9 & 25.6 & 0.05 \\
\hline Never reached full oral feeds & 25.7 & 42.3 & $0.03^{*}$ \\
\hline Death & 15.9 & 23.4 & 0.26 \\
\hline GT & 10 & 12.8 & 0.29 \\
\hline Combined death and GT & 26 & 36.4 & 0.21 \\
\hline Discharged with gavage feeds & 2.9 & 7.7 & 0.28 \\
\hline Combined death and discharged with gavage feeds & 17.4 & 31.2 & 0.06 \\
\hline $\mathrm{NEC}^{+}$ & 2.9 & 5.1 & 0.68 \\
\hline ECMO & 5.7 & 1.3 & 0.19 \\
\hline Elevated liver enzymes $^{\dagger}$ & 62.9 & 54.6 & 0.33 \\
\hline Elevated coagulation parameters ${ }^{\infty}$ & 100 & 80.4 & $0.0001^{*}$ \\
\hline Days to hospital discharge or death & $15.8 \pm 10.2$ & $16.2 \pm 10.9$ & 0.83 \\
\hline
\end{tabular}

* Significance defined by $P<0.05$.

${ }^{+}$Grossly bloody stool (Bell's stage IIA or greater).

${ }^{\dagger}$ Aspartate aminotransferase level $>200$ IU and alanine aminotransferase level $>100$ IU.

${ }^{\infty}$ Prothrombin time $>15.6$ seconds and partial thromboplastin time $>41.5$ seconds. 


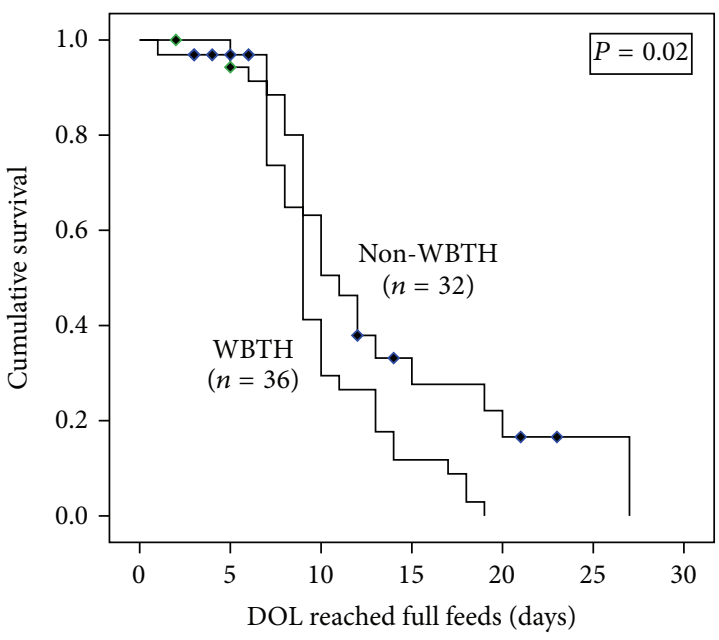

(a)

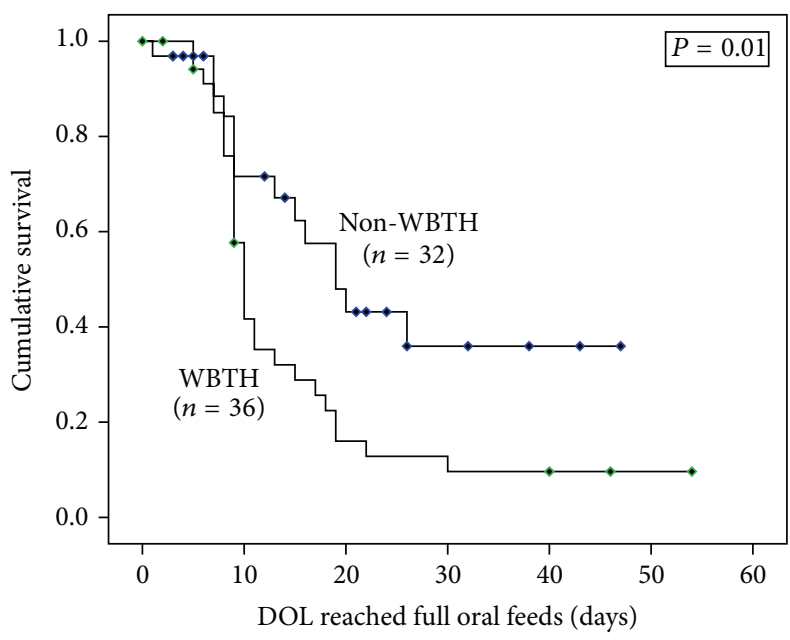

(b)

FIgURE 2: Survival curves for subjects with strict criteria ${ }^{*}$. Censored subjects are labeled.

primarily used for the initiation and advancement of feeds in preterm infants. There is no current standardized practice for initiating feeds in late-preterm and term infants with HIE at our institution. However, we expected to see a difference in practice for initiation of feeds over the twelve-year timeframe of our study, believing that many providers would feel more comfortable starting feeds sooner in the group of infants treated with WBTH. Since this was not the case, our results are actually strengthened by the fact that both groups were fed similarly.

Limitations of the study include the small sample size and retrospective analysis of data. A historical cohort study was performed because therapeutic hypothermia has been accepted as the standard of care for infants with moderate-tosevere HIE; a prospective analysis or randomized controlled trial is not an ethical option to evaluate the effects of WBTH on GI morbidity and feeding following a perinatal hypoxic event. We estimate that fewer infants with HIE were referred to our tertiary care institution prior to use of WBTH due to the lack of therapeutic options, further limiting the number of patients available for analysis. The study was also limited by the quality of documentation in the medical record. This was the reason for dividing the groups into strict and general criteria based on the level of documentation available. When there was a lack of adequate information, the research team either determined an infant that met criteria for moderateto-severe HIE based on other findings in the medical record (such as multiple abnormal lab values or even death) or decided to exclude the infant from the study if insufficient evidence was found. Although the 5 minute APGAR score was lower in the non-WBTH strict group versus the WBTH strict group, both groups had a mean score $<5$ at 5 minutes of life to suggest a considerable level of depression after birth in each one. The lower mortality seen specifically in the WBTH strict criteria group may arguably be explained by the fact that these infants were already a subgroup of infants with more significant illness. However, the lower mortality combined with more significant results seen in the groups of infants with clear evidence of moderate-to-severe HIE by a more strict definition validates the strict use of cooling criteria used by our institution.

It should be noted that a requirement for GT placement or gavage feeds at discharge or the number of days to reach full oral feeds could all be reflections of oral skills. Those differences seen in our study could arguably be similar to the known improvements in neurologic function seen with WBTH. However, advancement of enteral feeds is a more specific measure of GI motility and the decreased number of days to reach full enteral feeds alone suggests a nonneural benefit to WBTH.

Future implications for study may include use of therapeutic hypothermia for other causes of gastrointestinal ischemia-reperfusion injury such as NEC. NEC is a unique form of gastrointestinal ischemia that occurs most often in preterm infants. Its exact etiology is not fully elucidated and felt to be multifactorial. In 2010, Hall et al. [17] published a small randomized prospective pilot study establishing the safety of using WBTH in preterm infants with advanced stage surgical NEC. The authors of the study plan to complete a larger multicenter randomized controlled trial evaluating the use of therapeutic hypothermia to decrease the amount of GI ischemic injury and surgical intervention required for NEC. Although our study was not powered for the incidence of NEC, we see interesting trends in the small numbers of patients with NEC and moderate-to-severe HIE. Our study strongly supports the need for further research in this area. Next steps could include a larger, multicentered cohort study evaluating the incidence of NEC in infants with HIE before and after the use of WBTH.

In conclusion, WBTH use for moderate-to-severe HIE may have beneficial effects to the newborn that extend beyond neurocognitive and neurodevelopmental outcomes. 
Our findings suggest that WBTH improves GI morbidity and feeding tolerance for infants with moderate-to-severe HIE when strict criteria for cooling are applied.

\section{Conflict of Interests}

The authors declare no conflict of interests.

\section{References}

[1] D. V. Azzopardi, B. Strohm, A. D. Edwards et al., "Moderate hypothermia to treat perinatal asphyxial encephalopathy," The New England Journal of Medicine, vol. 361, no. 14, pp. 1349-1358, 2009.

[2] S. E. Jacobs, C. J. Morley, T. E. Inder et al., "Whole-body hypothermia for term and near-term newborns with hypoxic-ischemic encephalopathy: a randomized controlled trial," Archives of Pediatrics and Adolescent Medicine, vol. 165, no. 8, pp. 692700, 2011.

[3] S. Shankaran, A. R. Laptook, R. A. Ehrenkranz et al., "Whole-body hypothermia for neonates with hypoxic-ischemic encephalopathy," The New England Journal of Medicine, vol. 353, no. 15, pp. 1574-1584, 2005.

[4] S. Shankaran, A. Pappas, S. A. McDonald et al., "Childhood outcomes after hypothermia for neonatal encephalopathy," The New England Journal of Medicine, vol. 366, no. 22, pp. 20852092, 2012.

[5] H. Akinbi, S. Abbasi, P. L. Hilpert, and V. K. Bhutani, "Gastrointestinal and renal blood flow velocity profile in neonates with birth asphyxia," Journal of Pediatrics, vol. 125, no. 4, pp. 625-627, 1994.

[6] C. L. Berseth and H. H. McCoy, "Birth asphyxia alters neonatal intestinal motility in term neonates," Pediatrics, vol. 90, no. 5, pp. 669-673, 1992.

[7] A. Martin-Ancel, A. Garcia-Alix, F. Gaya, F. Cabanas, M. Burgueros, and J. Quero, "Multiple organ involvement in perinatal asphyxia," Journal of Pediatrics, vol. 127, no. 5, pp. 786-793, 1995.

[8] R. N. Goldberg, D. W. Thomas, and F. R. Sinatra, "Necrotizing enterocolitis in the asphyxiated full-term infant," The American Journal of Perinatology, vol. 1, no. 1, pp. 40-42, 1983.

[9] P. D. Gluckman, J. S. Wyatt, D. Azzopardi et al., "Selective head cooling with mild systemic hypothermia after neonatal encephalopathy: multicentre randomised trial," The Lancet, vol. 365, no. 9460, pp. 663-670, 2005.

[10] W. Zhou, G. Cheng, X. Shao et al., "Selective head cooling with mild systemic hypothermia after neonatal hypoxic-ischemic encephalopathy: a multicenter randomized controlled trial in China," Journal of Pediatrics, vol. 157, no. 3, pp. 367-372, 2010.

[11] A. Pierro and S. Eaton, "Intestinal ischemia reperfusion injury and multisystem organ failure," Seminars in Pediatric Surgery, vol. 13, no. 1, pp. 11-17, 2004.

[12] H. T. Hassoun, R. A. Kozar, B. C. Kone, H. J. Safi, and F. A. Moore, "Intraischemic hypothermia differentially modulates oxidative stress proteins during mesenteric ischemia/reperfusion," Surgery, vol. 132, no. 2, pp. 369-376, 2002.

[13] G. Stefanutti, A. Pierro, E. J. Parkinson, V. V. Smith, and S. Eaton, "Moderate hypothermia as a rescue therapy against intestinal ischemia and reperfusion injury in the rat," Critical Care Medicine, vol. 36, no. 5, pp. 1564-1572, 2008.
[14] H. B. Sarnat and M. S. Sarnat, "Neonatal encephalopathy following fetal distress: a clinical and electroencephalographic study," Archives of Neurology, vol. 33, no. 10, pp. 696-705, 1976.

[15] P. Shah, S. Riphagen, J. Beyene, and M. Perlman, "Multiorgan dysfunction in infants with post-asphyxial hypoxic-ischaemic encephalopathy," Archives of Disease in Childhood: Fetal and Neonatal Edition, vol. 89, no. 2, pp. F152-F155, 2004.

[16] S. Shankaran, E. Woldt, T. Koepke, M. P. Bedard, and R. Nandyal, "Acute neonatal morbidity and long-term central nervous system sequelae of perinatal asphyxia in term infants," Early Human Development, vol. 25, no. 2, pp. 135-148, 1991.

[17] N. J. Hall, S. Eaton, M. J. Peters et al., "Mild controlled hypothermia in preterm neonates with advanced necrotizing enterocolitis," Pediatrics, vol. 125, no. 2, pp. e300-e308, 2010. 


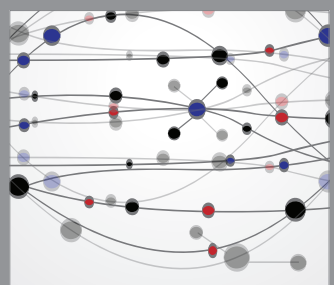

The Scientific World Journal
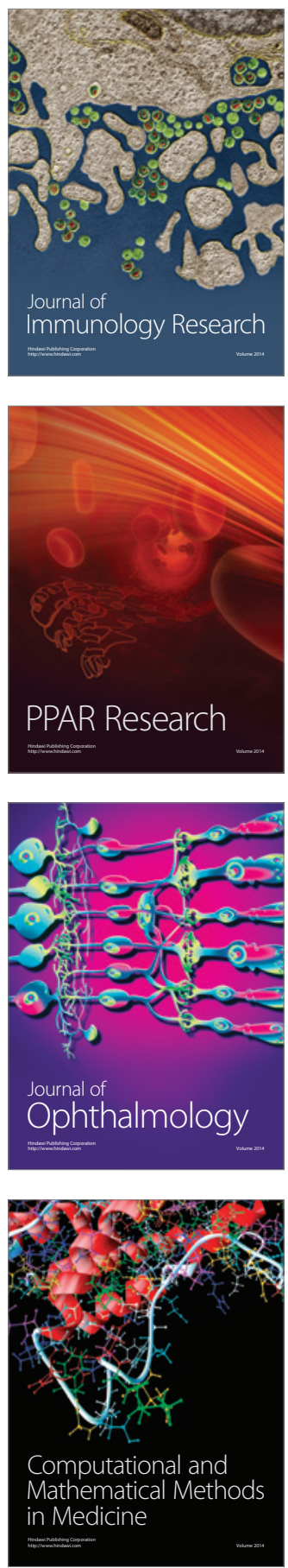

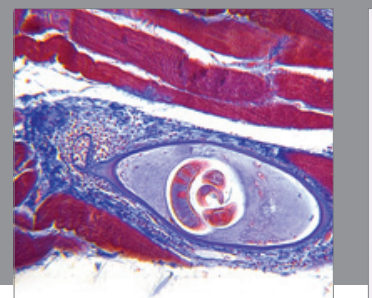

Gastroenterology

Research and Practice
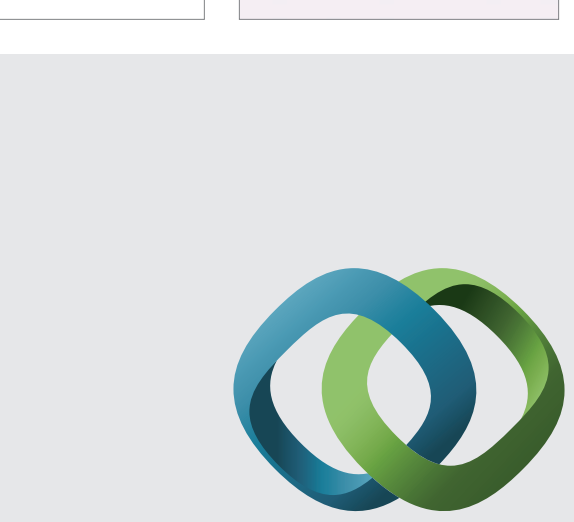

\section{Hindawi}

Submit your manuscripts at

http://www.hindawi.com
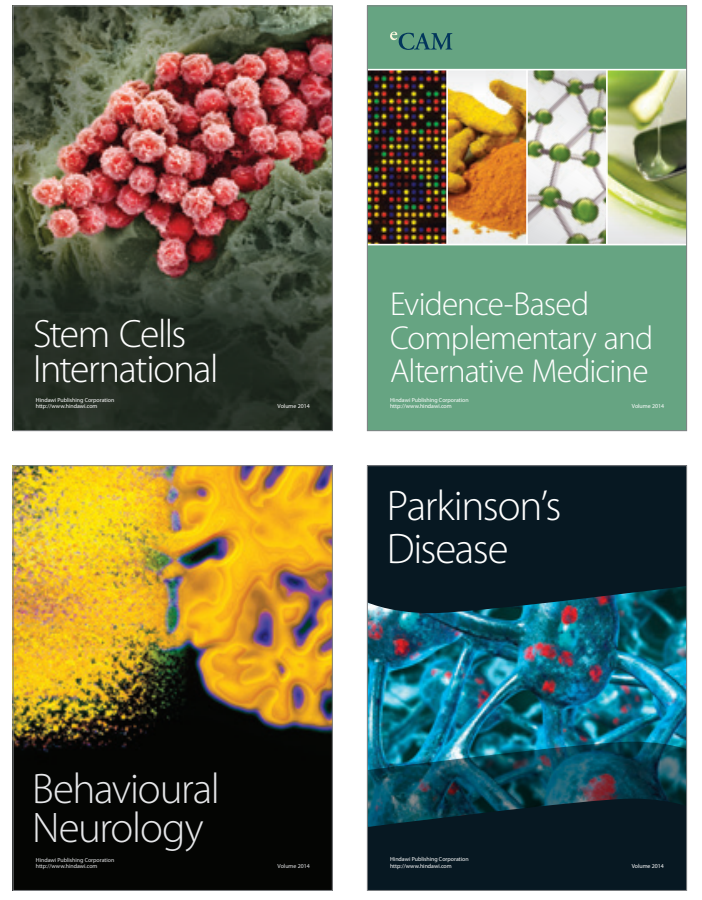
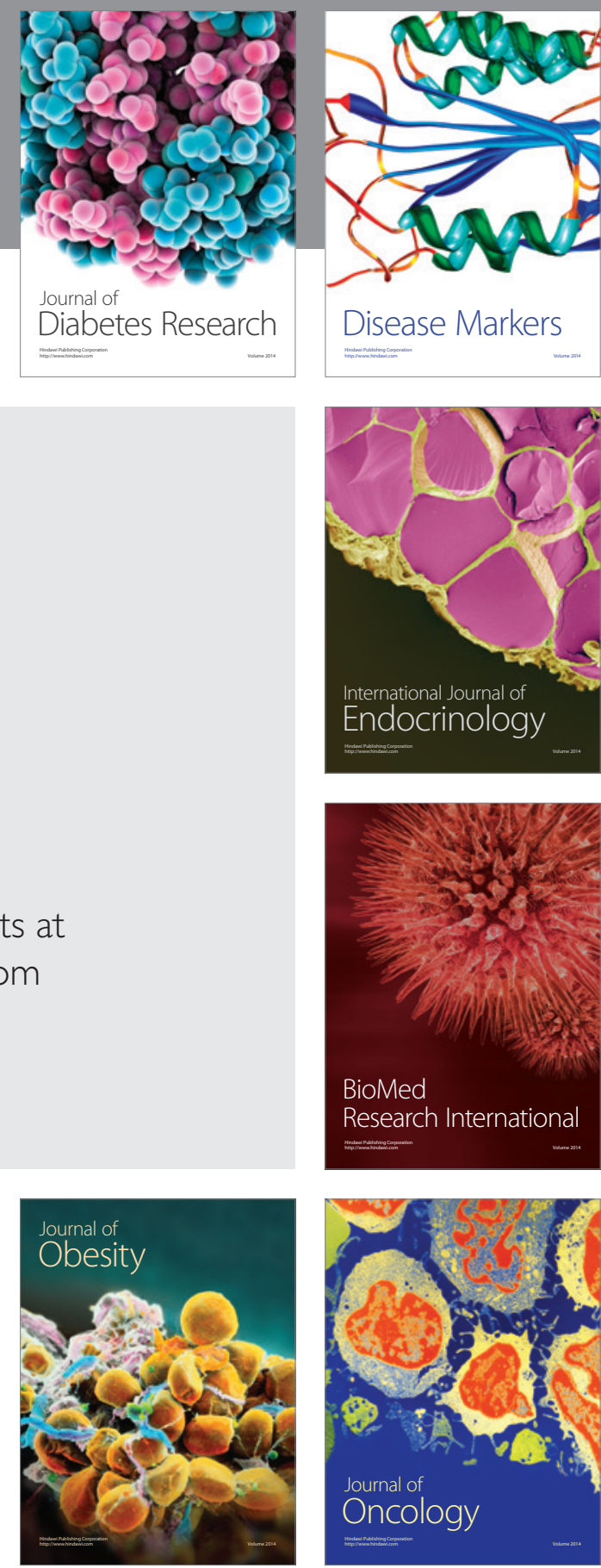

Disease Markers
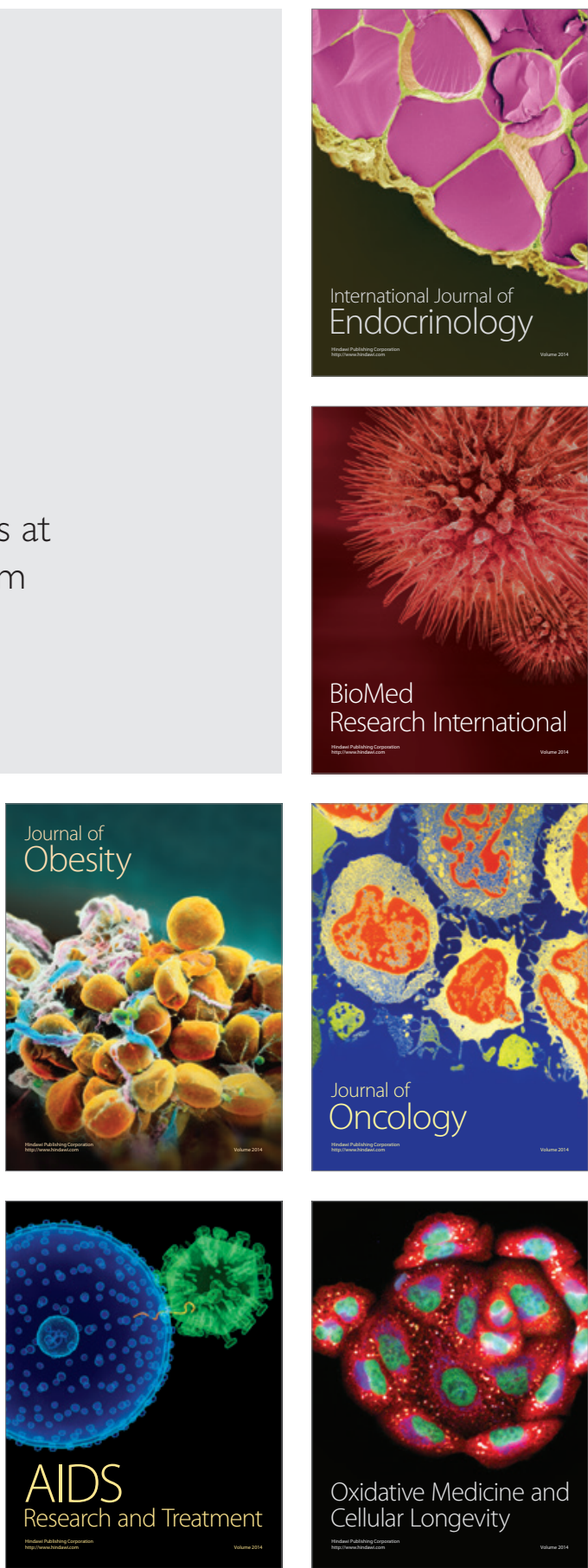